\title{
Public Health and Community Medicine
}

ORIGINAL ARTICLE

\section{Racial and Geographic Differences in Hospitalizations for Pediatric Opioid Use Disorders}

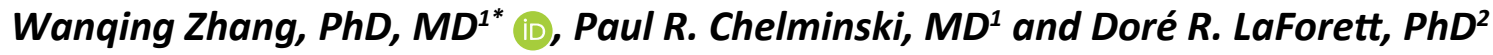 \\ ${ }^{1}$ Department of Allied Health Sciences, School of Medicine, University of North Carolina, North Carolina, USA \\ ${ }^{2}$ Frank Porter Graham Child Development Institute, University of North Carolina, North Carolina, USA
}

*Corresponding author: Wanqing Zhang, Department of Allied Health Sciences, School of Medicine, University of North Carolina at Chapel Hill, 321 S. Columbia Street, Chapel Hill, NC 27599-7122, USA, Tel: 919-962-4069

\begin{abstract}
Background: Opioids present unprecedented threat in the United States. Hospitalizations associated with opioid use disorders (OUD) among US children have doubled in the last decade. Important racial disparities in OUD have recently been noted. The purpose of this study is to explore the role of race in hospitalizations associated with OUD among children and adolescents across the 4 US Census Bureau-defined regions.
\end{abstract}

Methods: We performed a cross-sectional analysis of the US Nationwide Inpatient Sample involving child and adolescent inpatients. Multinomial logistic regression analyses were conducted to determine potential effects of race and region on the likelihood of child and adolescent inpatient stays for 3 OUD subtypes after controlling for demographic and clinical characteristics. The 3 OUD subtypes include: OUD-related hospitalization for opium dependency or abuse, OUD-related hospitalization for opium poisoning, and OUD-related hospitalization for external injury. The 4 US census regions include: Northeast, Midwest, South and West.

Results: Regional variations of association between race and OUD-related hospitalizations were found among child and adolescent inpatients. Hispanic children in South region were more likely to be hospitalized for reasons related to opium dependency or abuse than were white children in the same region (adjusted odds ratio 1.56, 95\% Cl 1.18-2.07). Overall, adolescent hospitalizations related to both opium dependency/abuse and opium poisoning were more likely to occur among white adolescents than among adolescents of other races. In 3 of the 4 US regions, black adolescents had higher odds of hospitalization for OUD-related external injury as compared to white adolescents (all $P<0.05$ ).

Conclusions: Significant racial and regional differences were found in OUD-related hospitalizations among children and adolescents. It is important to understand how geographic differences affect hospitalization outcomes for pediatric patients with OUD and to determine whether the use of inpatient care leads to improved outcomes for identifiable racial subgroups of children and adolescents in a specific geographical region. These findings may have important public health implications of the opioid epidemic. Policymakers should allocate resources to where they are most needed.

\section{Keywords}

OUD, Hospitalizations, Children and adolescents, Racial disparities, US Census Bureau-defined regions

\section{Abbreviations}

OUD: Opioid Use Disorders; HCUP: Healthcare Cost and Utilization Project; NIS: Nationwide Inpatient Sample; Cl: Confidence Interval

\section{Introduction}

The recent rise in pediatric opioid use in the United States is unprecedented, and exists in all pediatric age groups $[1,2]$. A national study showed that hospitalizations for pediatric opioid poisonings almost doubled from 1997 to 2012. The highest hospitalization rates occurred among older adolescents, while the largest increase in hospitalizations over time was seen in younger children [2]. The Agency for Healthcare Quality and Research (AHRQ) reported that opioid use disorders (OUD) are ranked among the top three substance use disorders resulting in hospital stay for teenagers [3]. Severe outcomes following opioid exposure were especially prevalent for young children [4-7].

Citation: Zhang W, Chelminski PR, LaForett DR (2020) Racial and Geographic Differences in Hospitalizations for Pediatric Opioid Use Disorders. Int Arch Public Health Community Med 4:042. doi. org/10.23937/2643-4512/1710042

Accepted: May 19, 2020; Published: May 21, 2020

Copyright: (C) 2020 Zhang W, et al. This is an open-access article distributed under the terms of the Creative Commons Attribution License, which permits unrestricted use, distribution, and reproduction in any medium, provided the original author and source are credited. 
Ethnic minorities are underrepresented in OUD research. A possible reason is that minorities have been reported to receive opioids less often for both adults and children [8,9]. Racial disparities exist in medical care and treatment associated with OUD [10]; for example, black and Hispanic adolescents with commercial insurance coverage have been found to be less likely than their white counterparts to receive medications for OUD [11]. Furthermore, regional difference in racial composition is an important factor when considering racial differences in OUD hospitalizations. When measuring health services utilization for children and adolescents geographic disparities are an important predictor. Such disparities are reflective of state policies and regional health care market characteristics (e.g., medical practice patterns and availability of services) [12].

To our knowledge, no study has explored racial differences in OUD hospitalizations in an inpatient pediatric population. This study intends to fill this research gap by examining the characteristics of pediatric hospitalizations related to OUD, with particular emphasis on the role of racial differences across US regions by 3 OUD subtypes according to the diagnostic codes at hospital discharge. The aim of this study was to explore the role of race in OUD-related pediatric hospitalizations across 4 US regions by analyzing the largest all-payer inpatient database in the US. We used nationally representative hospital discharge data from the publicly available Nationwide Inpatient Sample (NIS) to determine whether disparities exist across US regions among pediatric inpatients with different subtypes of OUD. We hypothesized that for both child and adolescent inpatients, hospitalization patterns vary by racial group and OUD subtypes in the 4 US regions, while taking into account the effect of clinical and other sociodemographic determinants. South region was the most diverse with the highest proportion of minority patients; thus, we expected that the variation in pediatric OUD hospitalizations would be greater in the South. Examining disparities in OUD-related hospitalizations could inform the development and implementation of public policies to promote evidence-based treatment across regions and pediatric populations affected by the opioid epidemic.

\section{Methods}

\section{Data source and study population}

Analysis was performed on secondary data from the 2012-2014 NIS. NIS is part of the Healthcare Cost and Utilization Project (HCUP) sponsored by the Agency for Healthcare Research and Quality (AHRQ). We pooled 3 years of data to improve the precision of estimates and to enable comparisons across subgroups. At the time of this study, the most recent 2015 NIS datasets include a mixture of ICD-9-CM and ICD-10-CM diagnosis codes; thus the 3-year period of 2012-2014 was selected to allow for analysis of a coherent set of variables with the same survey design methods [13]. In this analysis, our study sample was pediatric inpatients, including children and adolescents up to 21 years. Consistent with recommendations from the American Academy of Pediatrics [14], patients younger than 12 years were categorized as children, and patients between 12 to 21 years were categorized as adolescents.

\section{Variables and definitions}

All pediatric OUD were identified from the International Classification of Diseases, Ninth Revision, Clinical modification (ICD-9) in the NIS datasets. An OUD diagnosis was based on ICD-9 codes: 304.0x (opioid-type dependence), 304.7x (combinations of opioid-type drug dependence with any other drug dependence), 305.0x (nondependent opioid abuse), 965.00 (poisoning by opium), 965.01 (poisoning by heroin), 965.02 (poisoning by methadone), 965.09 (poisoning by other opiates and narcotics), and 970.1 (poisoning by opiate antagonists). Based on the recommendation from HCUP, ICD9-CM external cause of injury codes (E codes) were also included as representing OUD-related inpatient stays [15]. These E-codes include: E850.x (accidental poisoning by heroin; methadone; other opiates and related narcotics), E935.x (heroin, methadone, other opiates and related narcotics causing adverse effects in therapeutic use), and E940.1 (opiate antagonists causing adverse effects in therapeutic use). A hospitalization was coded as OUD-related if any of the above diagnostic codes were present among the 15 potential diagnoses. Furthermore, we created 3 subtypes of OUDs based on the above ICD-9 codes: OUD-related hospitalization for opium dependency or abuse (OUD subtype 1 , including 304.0x, 304.7x and 305.0x), OUD-related hospitalization for opium poisoning (OUD subtype 2, including 965.00, 965.01, 965.02, 965.09 and 970.1), and OUD-related hospitalization for external injury (OUD subtype 3 , including E850.x, E935.x and E940.1).

Race/ethnicity was classified into: Non-Hispanic whites, non-Hispanic blacks, Hispanics, and other racial minorities (Asian and all other races). The 4 US Census Bureau-defined regions included: Northeast, Midwest, South and West. In addition, we also used sex (male and female), primary insurance type (public, private, uninsured), and median household income of patient ZIP code $\left(0-25^{\text {th }}\right.$ percentile, $26^{\text {th }}$ to $50^{\text {th }}$ percentile, $51^{\text {st }}$ to $75^{\text {th }}$ percentile, and $76^{\text {th }}$ to $100^{\text {th }}$ percentile), patient disposition at the time of discharge (routine vs. others), hospital length of stay, severity of illness (minor/no comorbidity, moderate, and major/extreme), and hospital characteristics such as teaching status (teaching vs. nonteaching) and hospital bed size (small, medium, and large).

\section{Analysis}

Descriptive statistics for demographic and clinical variables were computed separately for child and ad- 
olescent inpatients. Categorical variables were summarized with the use of proportions and respective percentages. Continuous variables (age, hospital length of stay) were summarized using means with standard error (SE). A Chi-square test was used to compare categorical variables using the SURVEYFREQ procedure. Appropriate survey discharge weights were applied to the NIS data to obtain the national estimate. For both children and adolescents, we determined associations of demographic and clinical characteristics with the 3 subtypes of pediatric OUD using survey logistic regression as odds ratios (OR) and $95 \%$ confidence intervals $(95 \% \mathrm{Cl})$ adjusted for patient and hospital characteristics. We compared the characteristics for the 3OUD subtypes and for the non-OUD group using descriptive statistics. We then employed a multinomial logistic regression model, with the outcome being a four-level categorical variable (OUD subtype 1 , OUD subtype 2, OUD subtype 3 and non-OUD group). Multinomial regression was used to examine the different effects of the individual variables on the probability of OUD-related hospitalizations among 3 cohorts of pediatric patients in the OUD group within 4 regional strata with the dependent outcome of three OUD groups (the non-OUD group was used as the reference category). Domain analysis results from the SURVEYLOGISTIC procedure were obtained for the 4 US regions. A two-sided $p$ value $<0.05$ was used to determine statistical significance. Statistical analysis was performed using SAS 9.4 Software (SAS Institute Inc., Cary, NC, USA). Institutional Review Board (IRB) approval was not required as the NIS is a publicly available de-identified database.

\section{Results}

\section{Characteristics of the study sample}

The characteristics in OUD-related hospitalizations for children and adolescents are displayed in Table 1. There were substantial differences in demographic and

Table 1: Characteristics of OUD-related Hospitalizations among Children and Adolescents.

\begin{tabular}{|c|c|c|c|c|}
\hline & $\begin{array}{l}\text { Children } n(\%) \\
(n=9,895)\end{array}$ & P Value & $\begin{array}{l}\text { Adolescents } n(\%) \\
(n=93,020)\end{array}$ & P Value \\
\hline \multicolumn{5}{|l|}{ Variables } \\
\hline Age, mean (SE) & $3.5(0.06)$ & & $19.1(0.01)$ & \\
\hline Gender & & 0.0541 & & $<0.0001$ \\
\hline Male & $5,450(54.5)$ & & $45,830(49.3)$ & \\
\hline Female & $4,545(45.5)$ & & $47,185(50.7)$ & \\
\hline Race & & 0.0001 & & $<0.0001$ \\
\hline White & $4,955(55.8)$ & & $66,365(78.5)$ & \\
\hline Black & $1,395(15.7)$ & & $7,365(8.7)$ & \\
\hline Hispanic & $1,655(18.6)$ & & $6,550(7.8)$ & \\
\hline Other & $880(9.9)$ & & $4,250(5.0)$ & \\
\hline Insurance & & $<0.0001$ & & $<0.0001$ \\
\hline Public & $6,005(60.1)$ & & $38,080(41.1)$ & \\
\hline Private & $3,615(36.2)$ & & $43,895(47.3)$ & \\
\hline Uninsured & $380(3.8)$ & & $10,800(11.6)$ & \\
\hline Median household income & & 0.1357 & & $<0.0001$ \\
\hline $0-25^{\text {th }}$ percentile & $2,940(30.0)$ & & $20,890(23.0)$ & \\
\hline $26^{\text {th }}$ to $50^{\text {th }}$ percentile & $2,655(27.1)$ & & $21,850(24.1)$ & \\
\hline $51^{\text {st }}$ to $75^{\text {th }}$ percentile & $2,285(23.3)$ & & $22,285(24.5)$ & \\
\hline $76^{\text {th }}$ to $100^{\text {th }}$ percentile & $1,915(19.6)$ & & $25,815(28.4)$ & \\
\hline US Census region & & $<0.0001$ & & $<0.0001$ \\
\hline Northeast & $1,310(13.1)$ & & $24,845(26.7)$ & \\
\hline Midwest & $2,155(21.5)$ & & $22,145(23.8)$ & \\
\hline South & $4,130(41.2)$ & & $26,520(28.5)$ & \\
\hline West & $2,420(24.2)$ & & $19,510(21.0)$ & \\
\hline Hospital size & & $<0.0001$ & & $<0.0001$ \\
\hline Small & $1,880(18.8)$ & & $15,025(16.2)$ & \\
\hline Median & $2,270(22.7)$ & & $24,315(26.1)$ & \\
\hline Large & $5,865(58.6)$ & & $53,680(57.7)$ & \\
\hline Hospital teaching status & & $<0.0001$ & & $<0.0001$ \\
\hline
\end{tabular}




\begin{tabular}{|l|l|l|l|l|}
\hline Teaching & $8,800(87.9)$ & & $53,720(57.8)$ & \\
\hline Non-teaching & $1,215(12.1)$ & & $39,300(42.2)$ & $<0.0001$ \\
\hline Discharge status & & $<0.0001$ & & \\
\hline Routine & $8,385(83.8)$ & & $20,960(22.6)$ \\
\hline Other & $1,620(16.2)$ & & & $<0.0001$ \\
\hline APRDRG_Severity & & $<0.0001$ & $32,595(35.0)$ \\
\hline Minor/no comorbidity & $2,910(29.1)$ & & $43,865(47.2)$ \\
\hline Moderate & $2,725(27.2)$ & & $16,560(17.8)$ & \\
\hline Major/extreme & $4,380(43.7)$ & & $5.0(0.06)$ & \\
\hline Hospital LOS, mean (SE) & $13.6(0.53)$ & & \\
\hline
\end{tabular}

\section{- Opium dependency or abuse $\quad$ Opium poisoning OUD-related external injury}

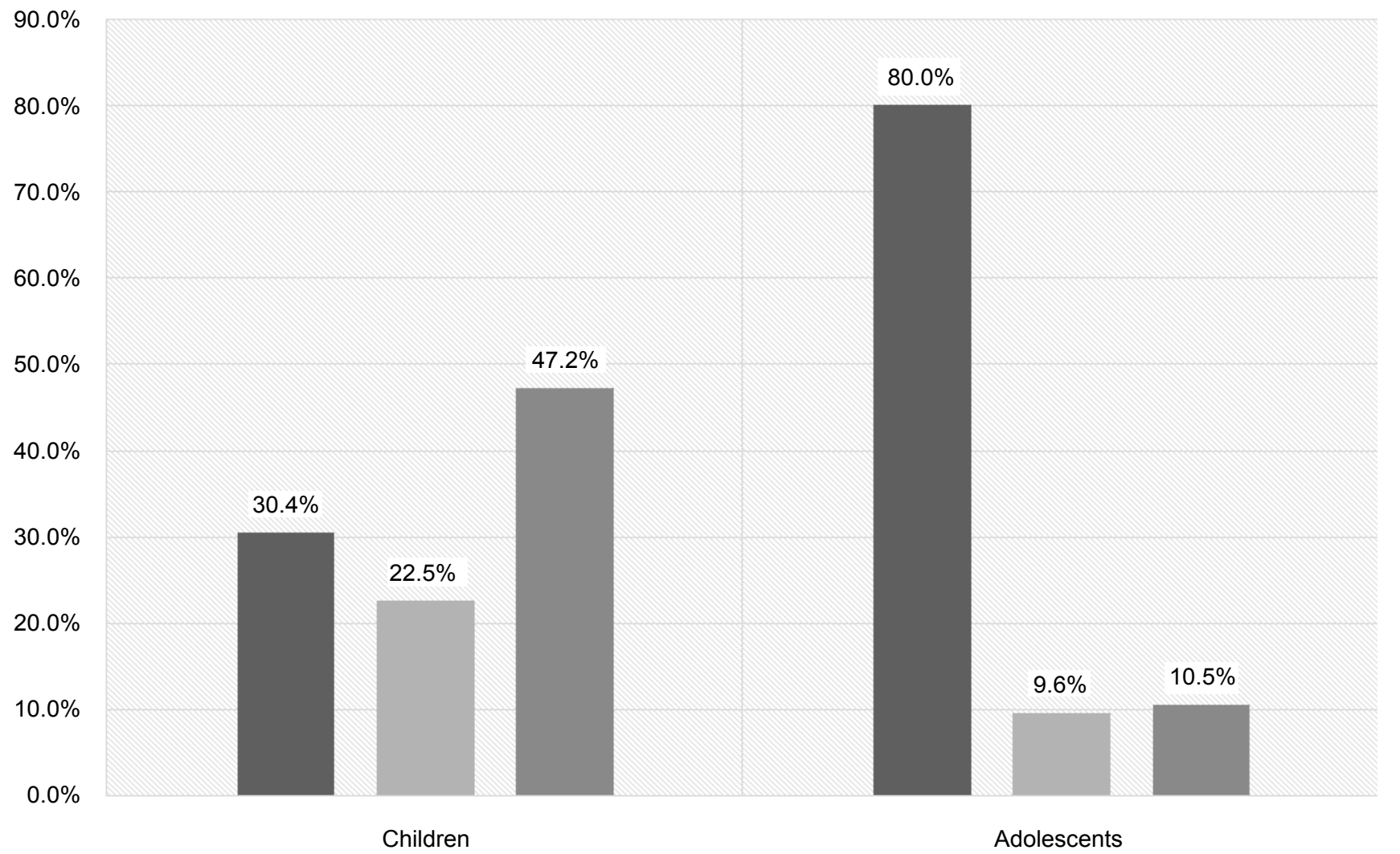

Figure 1: Distributions in Child and Adolescent Inpatients associated with an OUD Diagnosis by the 3 OUD Subtypes.

other characteristics of these pediatric inpatients. Child inpatients were more likely to be non-white and from an area with median household income below the $26^{\text {th }}$ percentile. Nearly $12 \%$ of adolescent inpatients were uninsured, compared to less than $4 \%$ of child inpatients. About two-thirds of child inpatients had public insurance. More than a quarter of adolescent inpatients were from the Northeast, while $41 \%$ of child inpatients were from the South. Nearly $90 \%$ of child inpatients were admitted to teaching hospitals, compared to $58 \%$ of adolescent inpatients. About $44 \%$ of child inpatients had major or extreme medical co-morbidities at hospital admission, compared to only $18 \%$ of adolescent inpatients. The length of hospital stay was almost tripled in child inpatients.
Figure 1 shows different distribution in child and adolescent inpatients associated with an OUD diagnosis by the 3 OUD subtypes. Among all OUD-related hospitalizations, it was notable that nearly half of child inpatients were hospitalized due to OUD-related external injury, compared to about $10 \%$ adolescent inpatients; three quarters of adolescent inpatients were hospitalized for opium dependency or abuse, compared to about onethird of child inpatients.

\section{Factors associated with OUD-related child and ad- olescent hospitalizations}

Table 2 shows the adjusted results of patient and clinical factors for OUD-related child and adolescent hospitalizations determined by multinomial logistic re- 
Table 2: Multivariable models of factors associated with OUD-related Pediatric Hospitalizations.

\begin{tabular}{|c|c|c|c|c|c|c|}
\hline & \multicolumn{2}{|c|}{$\begin{array}{l}\text { OUD subtype 1: } \\
\text { Opium dependency or abuse }{ }^{\dagger}\end{array}$} & \multicolumn{2}{|c|}{$\begin{array}{l}\text { OUD subtype } 2: \\
\text { Opium poisoning }\end{array}$} & \multicolumn{2}{|c|}{$\begin{array}{l}\text { OUD subtype } 3 \text { : } \\
\text { Opium-related external injury* }\end{array}$} \\
\hline & Children & Adolescents & Children & Adolescents & Children & Adolescents \\
\hline Variables & OR $(95 \% \mathrm{Cl})$ & OR $(95 \% \mathrm{Cl})$ & OR $(95 \% \mathrm{Cl})$ & OR $(95 \% \mathrm{Cl})$ & OR $(95 \% \mathrm{Cl})$ & OR $(95 \% \mathrm{Cl})$ \\
\hline Age & $1.22(1.19-1.24)$ & $1.46(1.45-1.47)$ & $1.14(1.11-1.16)$ & $1.08(1.06-1.10)$ & $1.28(1.26-1.30)$ & $0.96(0.94-0.97)$ \\
\hline Female vs. male & $0.95(0.80-1.13)$ & $0.43(0.41-0.45)$ & $1.03(0.84-1.25)$ & $0.61(0.55-0.68)$ & $1.00(0.87-1.15)$ & $0.79(0.72-0.88)$ \\
\hline Black vs. white & $0.57(0.44-0.75)$ & $0.21(0.19-0.22)$ & $0.64(0.49-0.85)$ & $0.44(0.37-0.52)$ & $0.91(0.75-1.11)$ & $1.49(1.33-1.67)$ \\
\hline Hispanic vs. white & $1.10(0.88-1.37)$ & $0.25(0.23-0.27)$ & $0.30(0.22-0.42)$ & $0.48(0.40-0.57)$ & $0.69(0.56-0.85)$ & $0.64(0.55-0.74)$ \\
\hline \multicolumn{7}{|c|}{ Public insurance vs. private insurance } \\
\hline & $1.22(1.00-1.49)$ & $0.94(0.91-0.98)$ & $2.75(2.13-3.54)$ & $0.73(0.65-0.82)$ & $1.01(0.86-1.18)$ & $0.71(0.64-0.78)$ \\
\hline
\end{tabular}

Uninsured vs. private insurance

\begin{tabular}{|l|l|l|l|l|l|}
$1.34(0.81-2.22)$ & $1.83(1.72-1.94)$ & $3.56(2.27-5.57)$ & $1.91(1.62-2.24)$ & $0.80(0.48-1.33)$ & $0.76(0.61-0.97)$ \\
\hline
\end{tabular}

Income quartile $0-25^{\text {th }}$ percentile vs. $76^{\text {th }}$ above

\begin{tabular}{|l|l|l|l|l|l|}
$1.04(0.79-1.35)$ & $0.56(0.53-0.60)$ & $1.27(0.90-1.78)$ & $0.82(0.70-0.97)$ & $0.90(0.72-1.13)$ & $0.75(0.65-0.87)$
\end{tabular}

Midwest vs. Northeast

\begin{tabular}{|l|l|l|l|l|l|}
$0.87(0.61-1.23)$ & $0.54(0.52-0.57)$ & $0.80(0.56-1.12)$ & $1.41(1.20-1.65)$ & $1.65(1.28-2.14)$ & $1.10(0.95-1.29)$
\end{tabular}

South vs. Northeast

\begin{tabular}{|l|l|l|l|l|l|}
$2.28(1.73-3.01)$ & $0.52(0.50-0.55)$ & $0.91(0.68-1.23)$ & $1.22(1.04-1.42)$ & $1.60(1.26-2.04)$ & $1.09(0.95-1.26)$
\end{tabular}

West vs. Northeast

\begin{tabular}{|l|l|l|l|l|l|}
$1.15(0.84-1.58)$ & $0.64(0.61-0.68)$ & $1.35(0.98-1.85)$ & $1.23(1.04-1.45)$ & $2.34(1.83-2.99)$ & $1.70(1.47-1.97)$
\end{tabular}

Large hospital vs. small

\begin{tabular}{|c|c|c|c|c|c|}
$0.59(0.47-0.74)$ & $0.84(0.79-0.88)$ & $1.52(1.09-2.11)$ & $0.87(0.75-1.00)$ & $0.73(0.60-0.89)$ & $0.92(0.81-1.06)$
\end{tabular}

Teaching hospital vs. non-teaching

\begin{tabular}{|l|l|l|l|l|l|}
$1.44(1.13-1.83)$ & $0.80(0.77-0.83)$ & $4.00(2.99-5.37)$ & $0.84(0.75-0.94)$ & $3.86(2.96-5.05)$ & $1.83(1.62-2.05)$
\end{tabular}

Discharge routine vs. non-routine

\begin{tabular}{|l|l|l|l|l|l|}
$0.46(0.37-0.57)$ & $0.41(0.39-0.43)$ & $0.99(0.60-1.62)$ & $0.15(0.13-0.17)$ & $0.68(0.55-0.84)$ & $0.77(0.66-0.89)$
\end{tabular}

APRDRG_Severity minor or no comorbidity vs. moderate

\begin{tabular}{|l|l|l|l|l|l|l}
$0.23(0.32-0.42)$ & $0.59(0.57-0.62)$ & $0.93(0.71-1.21)$ & $0.62(0.54-0.70)$ & $0.40(0.33-0.49)$ & $0.75(0.67-0.83)$
\end{tabular}

APRDRG_Severity major/extreme vs. moderate

\begin{tabular}{|l|l|l|l|l|l|}
$3.54(2.88-4.34)$ & $0.73(0.69-0.78)$ & $2.07(1.45-2.95)$ & $2.38(2.07-2.74)$ & $2.45(2.07-2.91)$ & $1.67(1.49-1.88)$
\end{tabular}

\begin{tabular}{|l|l|l|l|}
\hline $1.013(1.012-$ & & $1.013(1.011-$
\end{tabular}

\begin{tabular}{l|l|l|l|l|l|l|} 
Hospital LOS & $1.014)$ & $1.01(1.01-1.01)$ & $0.78(0.60-1.02)$ & $0.89(0.85-0.93)$ & $1.014)$ & $1.01(1.01-1.01)$ \\
\hline
\end{tabular}

Hospitalizations not involving an OUD diagnosis were specified as the baseline category.

†: Hospitalizations involving an OUD subtype 1 relative to those not involving an OUD diagnosis; ‡: Hospitalizations involving an OUD subtype 2 relative to those not involving an OUD diagnosis; ${ }^{*}$ : Hospitalizations involving an OUD subtype 3 relative to those not involving an OUD diagnosis.

gression models, using non-OUD hospitalizations as the reference group. Among child inpatients, being older, having public insurance, being treated in a teaching hospital, and having major medical co-morbidities were significantly associated with increased odds of all 3 OUD subtypes. Children in the South were more likely to be hospitalized for reasons related to opium dependency or abuse compared to children in the Northeast (adjusted OR 2.28, 95\% Cl 1.73-3.01); children in the West, Midwest and South regions were more likely to be hospitalized for OUD-related external injury compared to children in the Northeast (West: adjusted OR 2.34, 95\% $\mathrm{Cl} 1.83-2.99 ;$ Midwest: adjusted OR $1.65,95 \% \mathrm{Cl} 1.28-$ 2.14; South: adjusted OR 1.60, 95\% Cl 1.26-2.04).

Table 2 also provides the adjusted multivariable re- sults for adolescent inpatients. Overall, strong evidence suggests that adolescent hospitalizations related to both opium dependency/abuse and opium poisoning were more likely to occur among white adolescents than among adolescents of other races. However, black adolescents had greater odds of being hospitalized for OUD-related external injury (adjusted OR 1.49, 95\% Cl 1.33-1.67) as compared to white adolescents. Adolescent inpatients from the Northeast were more likely to be hospitalized for reasons related to opium dependency or abuse compared to adolescent inpatients in the other 3 regions. In addition, our data also revealed a statistically significant association between OUD-related adolescent hospitalizations and the following variables: Male gender, private insurance or uninsured, 
Table 3: Association between OUD Hospitalizations and Race by 4 US Regions.

\begin{tabular}{|c|c|c|c|c|}
\hline \multirow[b]{2}{*}{ Child Results } & Northeast & Midwest & South & West \\
\hline & $95 \% \mathrm{Cl}$ & OR $\quad 95 \% \mathrm{Cl}$ & OR $\quad 95 \% \mathrm{Cl}$ & OR $\quad 95 \% \mathrm{Cl}$ \\
\hline \multicolumn{5}{|c|}{ OUD Subtype 1 unadjusted } \\
\hline Black vs. White & $0.96 \quad 0.48-1.93$ & $1.120 .58-2.18$ & $0.68 \quad 0.49-0.96$ & $0.660 .24-1.85$ \\
\hline Hispanic vs. White & $0.88 \quad 0.42-1.81$ & $1.020 .40-2.58$ & $1.48 \quad 1.15-1.94$ & $0.770 .49-1.21$ \\
\hline \multicolumn{5}{|c|}{ OUD Subtype 1 adjusted ${ }^{*}$} \\
\hline Black vs. White & $0.730 .33-1.60$ & $0.70 \quad 0.36-1.37$ & $0.590 .42-0.84$ & $0.43 \quad 0.15-1.22$ \\
\hline Hispanic vs. White & $0.78 \quad 0.37-1.67$ & $0.88 \quad 0.34-2.28$ & $1.561 .18-2.07$ & $0.610 .37-0.98$ \\
\hline \multicolumn{5}{|c|}{ OUD Subtype 2 unadjusted } \\
\hline Black vs. White & $1.48 \quad 0.84-2.60$ & $0.960 .50-1.85$ & $0.83 \quad 0.56-1.24$ & $1.320 .67-2.58$ \\
\hline Hispanic vs. White & $0.35 \quad 0.13-0.98$ & $0.520 .16-1.68$ & $0.390 .22-0.69$ & $0.54 \quad 0.34-0.84$ \\
\hline \multicolumn{5}{|c|}{ OUD Subtype 2 adjusted } \\
\hline Black vs. White & $0.98 \quad 0.53-1.80$ & $0.46 \quad 0.22-0.97$ & $0.620 .41-0.93$ & $0.770 .40-1.51$ \\
\hline Hispanic vs. White & $0.20 \quad 0.07-0.58$ & $0.29 \quad 0.09-0.97$ & $0.33 \quad 0.18-0.59$ & $0.33 \quad 0.21-0.52$ \\
\hline \multicolumn{5}{|c|}{ OUD Subtype 3 unadjusted } \\
\hline Black vs. White & $0.99 \quad 0.57-1.73$ & $1.551 .08-2.23$ & $1.10 \quad 0.83-1.45$ & $\begin{array}{lll}0.77 & 0.41-1.43\end{array}$ \\
\hline Hispanic vs. White & $0.690 .36-1.31$ & $0.85 \quad 0.46-1.59$ & $0.64 \quad 0.45-0.91$ & $0.810 .60-1.08$ \\
\hline \multicolumn{5}{|c|}{ OUD Subtype 3 adjusted ${ }^{*}$} \\
\hline Black vs. White & $0.65 \quad 0.36-1.20$ & $1.15 \quad 0.77-1.73$ & $0.97 \quad 0.73-1.29$ & $0.57 \quad 0.31-1.07$ \\
\hline Hispanic vs. White & $0.49 \quad 0.25-0.98$ & $0.810 .43-1.55$ & $0.67 \quad 0.47-0.96$ & $0.720 .53-0.99$ \\
\hline \multicolumn{5}{|l|}{ Adolescent Results } \\
\hline \multicolumn{5}{|c|}{ OUD Subtype 1 unadjusted } \\
\hline Black vs. White & $0.13 \quad 0.11-0.15$ & $0.30 \quad 0.26-0.34$ & $0.16 \quad 0.14-0.19$ & $0.320 .26-0.38$ \\
\hline Hispanic vs. White & $0.20 \quad 0.16-0.23$ & $0.28 \quad 0.22-0.36$ & $0.13 \quad 0.11-0.15$ & $0.210 .19-0.23$ \\
\hline \multicolumn{5}{|c|}{ OUD Subtype 1 adjusted ${ }^{*}$} \\
\hline Black vs. White & $0.15 \quad 0.13-0.18$ & $0.30 \quad 0.26-0.35$ & $0.16 \quad 0.14-0.19$ & $0.35 \quad 0.29-0.42$ \\
\hline Hispanic vs. White & $0.25 \quad 0.22-0.29$ & $0.31 \quad 0.24-0.39$ & $0.18 \quad 0.15-0.22$ & $0.290 .26-0.33$ \\
\hline \multicolumn{5}{|c|}{ OUD Subtype 2 unadjusted } \\
\hline Black vs. White & $0.33 \quad 0.21-0.49$ & $0.46 \quad 0.35-0.62$ & $0.37 \quad 0.29-0.48$ & $0.26 \quad 0.14-0.48$ \\
\hline Hispanic vs. White & $0.25 \quad 0.15-0.41$ & $0.740 .50-1.09$ & $0.31 \quad 0.22-0.42$ & $0.350 .27-0.46$ \\
\hline \multicolumn{5}{|c|}{ OUD Subtype 2 adjusted } \\
\hline Black vs. White & $0.38 \quad 0.25-0.60$ & $0.610 .44-0.84$ & $0.43 \quad 0.34-0.56$ & $0.290 .15-0.53$ \\
\hline Hispanic vs. White & $0.330 .20-0.56$ & $0.87 \quad 0.59-1.28$ & $0.390 .28-0.55$ & $0.47 \quad 0.36-0.61$ \\
\hline \multicolumn{5}{|c|}{ OUD Subtype 3 unadjusted } \\
\hline Black vs. White & $1.491 .15-1.92$ & $1.261 .00-1.59$ & $1.491 .25-1.77$ & $0.93 \quad 0.68-1.27$ \\
\hline Hispanic vs. White & $0.57 \quad 0.39-0.84$ & $0.78 \quad 0.50-1.22$ & $0.490 .36-0.66$ & $0.47 \quad 0.38-0.58$ \\
\hline \multicolumn{5}{|c|}{ OUD Subtype 3 adjusted ${ }^{*}$} \\
\hline Black vs. White & $1.511 .14-2.01$ & $1.411 .08-1.83$ & $1.681 .41-2.01$ & $1.08 \quad 0.78-1.48$ \\
\hline Hispanic vs. White & $0.610 .41-0.92$ & $0.93 \quad 0.59-1.46$ & $0.50 \quad 0.37-0.69$ & $0.610 .49-0.77$ \\
\hline
\end{tabular}

*: Adjusted for age, sex, insurance status, median household income, hospital size, hospital teaching status, discharge status, APRDRG severity, and hospital LOS.

non-routine hospital discharges and median household income quartile above the $76^{\text {th }}$ percentile. For example, male sex was associated with higher likelihood of OUD-related hospitalizations among adolescent inpatients, whereas no significant differences were observed between sex and OUD-related hospitalizations among child inpatients. It is not able that a good portion of adolescent OUD inpatients were from high-income families with a median household income above the $76^{\text {th }}$ percentile, and the likelihood for OUD-related hospitalization increased for those having greater income; income was not a significant factor for child inpatients associated with an OUD diagnosis.

\section{Association between race and 3 OUD subtypes by US Regions}

Table 3 provides the unadjusted and adjusted results of multinomial logistic regression models stratified by 4 
US regions, showing the association between race and the 3 OUD-related hospitalization cohorts. Child and adolescent inpatients without an OUD diagnosis were used as the reference groups for all multinomial logistic regression models.

There were regional variations of association between race and OUD-related hospitalizations among child inpatients. Hispanic children in South region were more likely to be hospitalized for reasons related to opium dependency or abuse than were white children in the same region (OR 1.49, 95\% Cl 1.15-1.94; adjusted OR 1.56, 95\% Cl 1.18-2.07). In contrast, black children were less likely than were white children to be hospitalized for reasons related to opium dependency or abuse in the South (OR 0.68, 95\% Cl 0.49-0.96; adjusted OR $0.59,95 \% \mathrm{Cl} 0.42-0.84)$. The adjusted analysis also indicates that Hispanic children in West region were less likely to be hospitalized for reasons related to opium dependency or abuse when compared to white children in the West (adjusted OR 0.61, 95\% Cl 0.37-0.98). Among child inpatients with an opium poisoning diagnosis, results did not vary across US regions between Hispanic and white children, with higher adjusted ORs for white children across all 4 regions. In addition, unadjusted ORs of OUD-related external injury were higher for hospitalized black children in Midwest region as compared to white children in the same region, but there were no statistically significant differences after adjusting for patient and hospital characteristics.

The unadjusted and adjusted ORs were very similar among adolescent inpatients (Table 3). Regional stratified analysis indicates that white adolescent inpatients were consistently more likely to be hospitalized for opium abuse or dependency and opium poisoning than both black and Hispanic adolescent inpatients across 4 US regions. However, in 3 of the 4 US regions, black adolescent inpatients had a significantly higher likelihood of being hospitalized for OUD-related external injury than their white counterparts (Northeast: adjusted OR $=1.51,95 \% \mathrm{Cl}$ 1.14-2.01; Midwest: adjusted OR = 1.41, $95 \% \mathrm{Cl} 1.08-1.83$; South: Adjusted OR $=1.68,95 \% \mathrm{Cl}$ 1.41-2.01).

\section{Discussion}

Our findings revealed that important racial and regional differences exist in the subtypes of OUD-related hospitalizations among child and adolescent inpatients. When the non-OUD inpatient group is used for comparison, black adolescents had greater odds of being hospitalized for OUD-related external injury than did white adolescents. Compared to their white counterparts, Hispanic child inpatients had a higher odds ratio for hospitalizations related to opium dependency or abuse in the South. In addition, we found that the odds ratios of hospitalizations for OUD-related external injury were the highest in West region among both child and adolescent inpatients. In the Northeast, odds of hospitalizations related to opium dependency or abuse were significantly higher among adolescent inpatients than among their counterparts in the other 3 regions. These regional variations may reflect variations in access to health care providers, regional disparities in the use of prescription opioids, and hospital discharge rate. According to the AHRQ, when adjusted for the population of each region, hospitalizations related to abuse of any drug were most likely to occur in the Northeast [16].

Furthermore, our study shows that among adolescent inpatients, all 3 subtypes of OUD-related hospitalizations are related to the family income quartile. We found that nearly one-third of OUD adolescent inpatients were from a zip code area with the highest median household income. In addition, the treatment for most adolescent inpatients associated with opium dependency or abuse diagnoses was paid through private insurers. Previous research indicates that adolescents from affluent neighborhoods are at increased risk for substance abuse because there could be less parental supervision in these areas and more exposure to peers who abuse substances [17]. A national survey showed that of a quarter of high school seniors had used prescription opioids [18]. Allen, et al. found that serious medical outcomes from prescription opioid exposures were highest among teenagers [19]. A recent national study indicated that 1 in 1,600 privately insured adolescents had a prescription opioid overdose needing medical attention [20]. It is essential to limit access of prescription opioids by children and adolescents, as overdose and addiction are potential complications of receiving prescription opioids. Therefore, safe opioid prescription practices could be a critical strategy for addressing this issue.

Our study provides a unique picture of racial differences of OUD-related pediatric hospitalizations across 4 US regions. In South region, it is notable that Hispanic child inpatients were at higher odds for hospitalizations associated with a diagnosis of opium dependency or abuse as compared to their white counterparts. Young children are more vulnerable to accidental ingestion of opioids when the child is exposed to a drug prescribed for a parent or other adult in the household [6,21]. Finkelstein, et al. study showed that young children with opioid overdose were far more likely to have a mother who had received a prescription opioid [21]. Pediatric providers need to discuss the importance of safe medication storage with guardians [22]. Practitioners should advise parents or guardians on prescribed opioids to keep these medications away from their children's reach. Furthermore, specific treatment or public health intervention programs such as social rehabilitation, parental coaching and mentoring, and parental skills taught through cognitive behavioral therapy should be explored $[23,24]$. Hispanic families in South region may be particularly vulnerable due to their lack of resources and subsequent lack of access to such programs 
and services. This finding runs counter to the common misperception that the opioid epidemic is predominantly a white problem. Policy makers need to consider allocating resources where they are most needed.

Important racial disparities in prevalence and outcomes from opium use have recently been noted, indicating heightened risk of opioid use frequency and adverse health consequences due to opioid abuse and dependence among blacks and Hispanics. Racial disparities exist in opioid-related medical care [8]. Our results further highlight existing racial/ethnic disparities in OUD-related hospital utilizations among pediatric inpatients. Since the likelihood of hospitalizations for OUD-related external injury was significantly higher among black adolescents, appropriate inpatient care needs to consider cultural and ethnic factors. A better understanding of this dynamic could improve inpatient services by suggesting effective culturally responsive strategies to health care providers.

\section{Limitations}

There are some limitations to this analysis. The NIS dataset lacks specific practice-level or family-level information (e.g., practice-style variation at the regional level, parental influence) that could potentially confound the associations of race and OUD-related pediatric hospitalizations across US regions. In addition, the NIS data were originally collected for billing purposes and therefore have inherent limitations such as misclassification and inadequacy of information. Another limitation is that due to the de identified nature of the data, the unit of analysis is the hospital discharge, not a patient. Finally, we acknowledge that the broad geographic areas may limit the inferences that can be made in this analysis. Despite these limitations, to our knowledge, this is the first study examining hospitalization patterns among pediatric OUD nationally by race and US Census Bureau-defined regions. The current public health crisis stemming from prescription opioids is a systemic issue that affects children and adolescents of diversified racial groups across US regions. There is a critical need to better characterize the opioid epidemic in all groups, and especially in the pediatric population where data have been sparse. Therefore, the findings of this study improve the literature on racial disparities among children and adolescents with OUD.

\section{Conclusions and Public Health Implications}

Results from our large, nationally representative study suggest that it is important to examine the association between race and OUD-related hospitalizations by OUD subtypes across US regions. Racial disparities in OUD-related health services utilization and outcomes exist but remain understudied. The opioid epidemic is more of a white problem. Our study demonstrates that at a regional level, there are significant racial differences in pediatric OUD hospitalizations among children and adolescents. Racial disparities and regional variations may reflect variations in access to health care providers and regional population structure. The current public health crisis stemming from the misuse of an addiction to opioids is a systemic issue that affects children and adolescents of diversified racial groups across US regions. Our findings have implications for refocusing public policy to understanding core public health perceptions of the opioid epidemic, and provide a rationale for further exploration of key factors associated with improved outcomes and care for OUD for pediatric inpatients from different racial groups.

\section{Acknowledgements}

This project is supported by the Health Resources and Services Administration (HRSA) of the U.S. Department of Health and Human Services (HHS) under R40MC32880. The information, content and/or conclusions are those of the author and should not be construed as the official position or policy of, nor should any endorsements be inferred by HRSA, HHS or the U.S. Government. The authors would like to acknowledge the Healthcare Cost and Utilization Project (HCUP) for supplying the data for this analysis. The authors have no financial relationships or conflict of interest relevant to this article to disclose.

\section{Funding Source}

This study was supported by the Health Resources and Services Administration (HRSA) of the U.S. Department of Health and Human Services (HHS) under R40MC32880.

\section{Author Contributions}

Dr. Zhang conceived of the study, participated in its design and coordination, drafted the manuscript, and performed the statistical analysis; Drs. Chelminski and LaForett helped conceptualize the study and critically reviewed the manuscript for important intellectual content; all authors approved the final manuscript as submitted and agree to be accountable for all aspects of the work.

\section{References}

1. Coben JH, Davis SM, Furbee PM, Sikora RD, Tillotson RD, et al. (2010) Hospitalizations for poisoning by prescription opioids, sedatives, and tranquilizers. Am J Prev Med 38: 517-524.

2. Gaither JR, Leventhal JM, Ryan SA, Camenga DR (2016) National trends in hospitalizations for opioid poisonings among children and adolescents, 1997 to 2012. JAMA Pediatrics 170: 1195-1201.

3. Heslin KC, Elixhauser A (2016) Mental and substance use Disorders among hospitalized teenagers, 2012. HCUP Statistical Brief \#202. Agency for Healthcare Research and Quality, Rockville, MD.

4. Lovegrove MC, Weidle NJ, Budnitz DS (2015) Trends in emergency department visits for unsupervised pediatric medication exposures, 2004-2013. Pediatrics 136: e821-e829. 
5. Kim HK, Smiddy M, Hoffman RS, Nelson LS (2012) Buprenorphine may not be as safe as you think: a pediatric fatality from unintentional exposure. Pediatrics 130: e1700-e1703.

6. Bailey JE, Campagna E, Dart RC (2009) The under recognized toll of prescription opioid abuse on young children. Ann Emerg Med 3: 419-424.

7. Lovegrove MC, Mathew J, Hampp C, Governale L, Wysowski DK, et al. (2014) Emergency hospitalizations for unsupervised prescription medication ingestions by young children. Pediatrics 134: e1009-e1016.

8. Singhal A, Tien Y, Hsia RY (2016) Racial-ethnic disparities in Opioid prescriptions at emergency department visits for conditions commonly associated with prescription drug abuse. PLoS One 11: e0159224.

9. McCabe SE, West BT, Veliz P, McCabe VV, Stoddard SA et al. (2017) Trends in medical and nonmedical use of prescription opioids among US adolescents: 1976-2015. Pediatric 139: e20162387.

10. Santaro TN, Santaro JD (2018) Racial bias in the US opioid epidemic: review of the history and implications for care. Cureus 10: e3733.

11. Hadland SE, Wharam JF, Schuster MA, Zhang F, Samet $\mathrm{JH}$, et al. (2017) Trends in receipt of buprenorphine and naltrexone for opioid use disorder among adolescents and young adults, 2001-2014. JAMA Pediatrics 171: 747-755.

12. Sturm R, Ringel JS, Andreyeva T (2003) Geographic disparities in children's mental health care. Pediatrics 112: e308.

13. https://www.hcup-us.ahrq.gov/nisoverview.jsp

14. Hardin AP, Hackell JM (2017) Age limit of pediatrics. Pediatrics 140: e20172151.

15. Weiss AJ, Bailey MK, O'Malley L, Barrett ML, Elixhauser A, et al. (2017) Patient residence characteristics of opioid-re- lated inpatient stays and emergency department visits nationally and by State, 2014. HCUP Statistical Brief \#226. Agency for Healthcare Research and Quality, Rockville, MD.

16. Kassed CA, Levit KR, Hambrick MM (2007) Hospitalizations related to drug abuse, 2005. HCUP Statistical Brief \#39. Rockville, MD.

17. Trim RS, Chassin L (2008) Neighborhood socioeconomic status effects on adolescent alcohol outcomes using growth models: exploring the role of parental alcoholism. J Stud Alcohol Drugs 69: 639-648.

18. McCabe SE, West BT, Teter CJ, Boyd CJ (2012) Medical and nonmedical use of prescription opioids among high school seniors in the United States. Arch Pediatr Adolesc Med 166: 797-802.

19. Allen JD, Casavant MJ, Spiller HA, Chounthirath T, Hodges NL, et al. (2017) Prescription opioid exposures among children and adolescents in the United States: 2000-2015. Pediatrics 139: e20163382.

20. Groenewald CB, Zhou C, Palermo TM, Van Cleve WC (2019) Associations between opioid prescribing patterns and overdose among privately insured adolescents. Pediatrics 144: e20184070.

21. Finkelstein $Y$, Macdonald EM, Gonzalez A, Sivilotti MLA, Mamdani MM, et al. (2017) Overdose risk in young children of women prescribed opioids. Pediatrics 139: e20162887.

22. McDonald EM, Kennedy-Hendricks A, McGinty EE, Shields WC, Barry CL, et al. (2017) Safe storage of opioid pain relievers among adults living in households with children. Pediatrics 139: e20162161.

23. Yule AM, Lyons RM, Wilens TE (2018) Opioid use disorders in adolescents-updates in assessment and aanagement. Curr Pediatr Rep 6: 99-106.

24. (2019) Intervention IDEAs for Infants, Toddlers, Children, and Youth Impacted by Opioids. 